\title{
The effect of RAFT polymerization on the physical properties of thiamphenicol-imprinted polymer
}

\author{
Noor Fadilah Yusof, ${ }^{1}$, Faizatul Shimal Mehamod, ${ }^{2,}$, and Faiz Bukhari Mohd Suah ${ }^{3}$ \\ ${ }^{1}$ School of Fundamental Science, Universiti Malaysia Terengganu, 21030 Kuala Nerus, Malaysia \\ ${ }^{2}$ Advanced Nano Materials (ANoMA) Research Group, School of Fundamental Science, Universiti Malaysia Terengganu, 21030 \\ Kuala Nerus, Terengganu, Malaysia \\ ${ }^{3}$ School of Chemical Sciences, Universiti Sains Malaysia (USM), 11800 Minden, Pulau Pinang, Malaysia.
}

\begin{abstract}
The necessity to overcome limitation of conventional free radical polymerization, technology has shifted the way to find an effective method for polymer synthesis, called controlled radical polymerization (CRP). One of the most studied controlled radical system is reversible additionfragmentation chain transfer (RAFT) polymerization. The method relies on efficient chain-transfer processes which are mediated typically by thiocarbonyl-containing RAFT agents e.g., dithioesters. The presented study revealed the potential benefit in applying RAFT polymerization towards the synthesis of molecularly imprinted polymer for thiamphenicol. They were synthesized in monolithic form using methacrylic acid, ethylene glycol dimethacrylate, azobisisobutyronitrile and acetonitrile as a functional monomer, cross-linker, initiator and porogen, respectively. The surface morphology was studied by scanning electron microscopy (SEM), structural characterization by Fourier transformed infrared (FTIR) and pore structures of polymers produced were characterized by nitrogen sorption porosimetry. SEM analysis showed MIPs produced by RAFT have smoother surface while porosity analysis showed the specific surface area was slightly larger compared to conventional polymerization methods. However FTIR showed the same pattern of spectra produced due to the same co-monomers used in the production. The results upon the uses of RAFT polymerization enables the production of imprinted polymers enhanced the physical properties compared to conventional polymerization.
\end{abstract}

\section{Introduction}

Molecular imprinting is a facile and versatile approach for the generation of synthetic receptors with tailor-made recognition sites [1]. Molecularly Imprinted Polymers (MIPs) are normally prepared by conventional free radical polymerization (FRP) due to the tolerance of FRP for a wide range of functional groups in the monomers and templates, but also because conventional FRP can normally be carried out in a facile manner under mild reaction conditions. However, conventional FRP allows for only limited control over the polymer growth processes with regard to chain propagation and termination, as well as the chemical structures of the polymeric products $[2,3]$ plus polymer networks with heterogeneous structures are normally produced when such networks are synthesised using FRP.

The necessity to overcome these limitations urged synthetic polymer chemists to develop new concepts, which would permit for the preparation of MIPs with more homogeneous network structures, a better understanding of the structure-property relationship of MIPs, and for obtaining MIPs with improved binding properties [1]. In this respect, controlled/living radical polymerization (CRP) techniques have been evolved and it is well understood that CRP processes offer many benefits $[4,5]$

Reversible addition-fragmentation chain transfer (RAFT) is one of the most versatile ways to confer "living" characteristics onto radical polymerization [6]. As an alternative to conventional FRP for the production of MIPs, our hypothesis was that the controlled nature of 'living' radical polymerization would translate into MIPs with properties superior to those displayed by MIPs prepared by conventional FRP, e.g., improved homogeneity of binding sites and enhanced chromatographic performance.

The aim of this work was to explore the potential benefits in applying RAFT polymerization techniques towards the synthesis of MIPs, with thiamphenicol as a model template. In the present study, polymers were prepared via conventional free radical polymerization and controlled radical polymerization in the form of polymer monolith. The RAFT agent used was 2-(2'cyanopropyl)dithiobenzoate (CPDB). CPDB was selected as the RAFT agent because it has been used previously for the successful polymerization of methacrylates and styrenes $[7,8]$.The physical properties of these polymers were studied using SEM and

* Corresponding author: fshimal@,umt.edu.my 
porosimetry analysis by Brunauer-Emmett-Teller (BET) technique.

\section{Experimental and Method}

\subsection{Synthesis of thiamphenicol-imprinted polymer}

Methacrylic acid (MAA), 2,2'-azobisisobutyronitrile (AIBN), Thiamphenicol, ethylene glycol dimethacrylate (EGDMA) and 2-(2'-cyanopropyl)dithiobenzoate (CPDB RAFT agent) were purchased from Sigma Aldrich. Toluene and Acetonitrile were purchased from Fisher Scientific. All chemicals were purified and AIBN was re-crystallized by methanol before used.

The synthesis of the polymers was based upon a procedure reported by [9-11]. Thiamphenicol (0.5 mmol), MAA (2.3 mmol), EGDMA (11.6 mmol) and AIBN $(0.76 \mathrm{mmol})$ were dissolved in acetonitrile $(4 \mathrm{~mL})$ in a thick-walled glass Kimax culture tube together with CPDB $(1.5 \mathrm{mmol})$. The solution was deoxygenated by sparging with oxygen-free nitrogen for 10 minutes while cooling in an ice-bath. The tube was sealed under nitrogen by means of a screw-cap and placed in an oilbath for 48 hours with the temperature maintained at 60 ${ }^{\circ} \mathrm{C}$. The thiamphenicol-imprinted polymer, P1 MIP, was obtained as a monolith; the monolith was subsequently crushed, mechanically ground and wet-sieved using acetone. Particles of $<25 \mu \mathrm{m}$ were collected after sedimentation $(3 \mathrm{x})$ from acetone. In order to remove traces of unreacted monomers and the template, the polymer was extracted overnight in a Soxhlet apparatus using methanol, and then dried at $40{ }^{\circ} \mathrm{C}$ under vacuum. A non-imprinted control polymer (NIP), P2_NIP, was prepared in the same manner as P1_MIP but in the absence of thiamphenicol.

The thiamphenicol-imprinted polymer synthesised via conventional free radical polymerization, P3_MIP, was prepared in the same manner as P1_MIP but in the absence of CPDB. A non-imprinted control polymer, P4_NIP, was prepared in the absence of both CPDB and thiamphenicol.

Table 1. Bulk Polymerization.

\begin{tabular}{|c|c|c|c|c|}
\hline Polymers & P1_MIP & P2_NIP & P3_MIP & P4_NIP \\
\hline $\begin{array}{c}\text { Thiamphenicol } \\
(\mathrm{mmol})\end{array}$ & 0.5 & - & 0.5 & - \\
\hline $\begin{array}{c}\text { MAA } \\
(\mathrm{mmol})\end{array}$ & 2.3 & 2.3 & 2.3 & 2.3 \\
\hline $\begin{array}{c}\text { EGDMA } \\
(\mathrm{mmol})\end{array}$ & 11.6 & 11.6 & 11.6 & 11.6 \\
\hline $\begin{array}{c}\text { Solvent } \\
(\mathrm{ml})\end{array}$ & 4 & 4 & 4 & 4 \\
\hline $\begin{array}{c}\text { AIBN } \\
(\mathrm{mmol})\end{array}$ & 0.76 & 0.76 & 0.76 & 0.76 \\
\hline $\begin{array}{c}\text { CPDB } \\
(\mathrm{mmol})\end{array}$ & 1.5 & 1.5 & - & - \\
\hline
\end{tabular}

\subsection{Physical characterizations}

All polymers obtained were characterized by using Scanning Electron Microscopy (SEM) model JEOL JSM-6360LA. The nitrogen gas adsorption method were applied using surface area and porosity analyzer (ASAP 2020 V4.02) manufactured by Micromeritics. This instrument operates by adsorp the nitrogen gas on the surface of polymer samples at $77 \mathrm{~K}$. Each sample was degassed at $150{ }^{\circ} \mathrm{C}$ for 8 hours before measurement. The data obtained were evaluated though the techniques of Brunauer-Emmett-Teller (BET) to calculate the specific surface area and Barret-Joyner-Halenda (BJH) for specific pore volume.

\section{Results and Discussion}

\subsection{Synthesis of thiamphenicol-imprinted polymer}

The MIPs (P1_MIP and P3_MIP) and NIPs (P2_NIP and P4_NIP) were synthesised successfully in good yield and the form of monoliths, using two different polymerization approaches: RAFT polymerization (P1_MIP and P2_NIP) and conventional FRP (P3_MIP and $\mathrm{P} 4$ NIP). Fig. 1 shows the chemical structure of $\mathrm{CPDB}$, thiamphenicol, MAA and EGDMA.

The polymers were synthesised on a $4.0 \mathrm{~g}$ monomer scale. Polymers P1_MIP (3.107g, 78\%) and P2_NIP $(3.012 \mathrm{~g}, 75 \%)$ were obtained as pink/purple coloured optically-transparent monoliths whereas P3 MIP (2.876g, 72\%) and P4 NIP (3.194g, 80\%) were obtained as white, opaque monoliths.<smiles>CC(C)(C#N)SC(=S)c1ccccc1</smiles>

(a)<smiles>CS(=O)(=O)c1ccc(C(O)C(CO)NC(=O)C(Cl)Cl)cc1</smiles>

(b)<smiles>C=C(C)C(=O)O</smiles>

(c)<smiles>C=C(C)C(=O)OCCOC(=O)C(=C)C</smiles>

(d)
Fig. 1. Chemical structures of (a) the RAFT agent (CPDB), (b) template (thiamphenicol), (c) functional monomer (methacrylic acid; MAA) and (d) crosslinking agent (ethylene glycol dimethacrylate; EGDMA) exploited in this study

\subsection{Physical characterizations}

The size and shape of the various polymer particles were analysed by SEM. As expected for polymer particles produced through the mechanical grinding of monoliths, the particles obtained were irregularly shaped. The particle sizes were defined by the grinding, sieving and 
sedimentation processes. Only particles with sizes of $<25$ $\mu \mathrm{m}$ were collected and used in this study. P1_MIP and P2_NIP had the typical appearance of a gel-type polymer when in the dry state and they were optically transparent (Fig. 2). In contrast, P3_MIP and P4_NIP were scattered white light, suggestive of well-developed pore structures (Fig. 3). The fact that the presence of RAFT agent in the P1_MIP had a profound impact upon the morphology of the product [9]. These observations were confirmed by nitrogen sorption porosimetry experiments which will explain further.

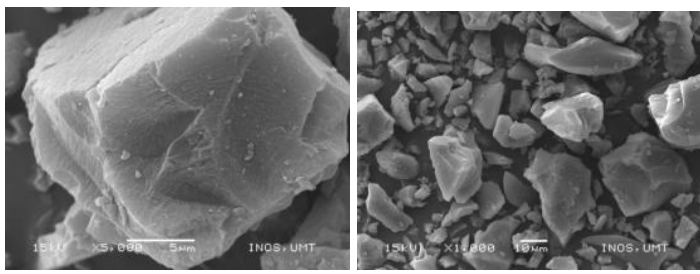

(a)

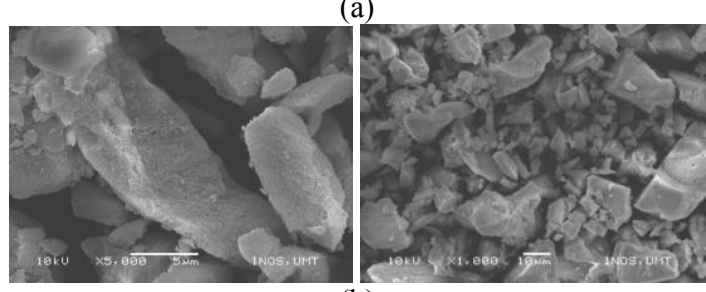

(b)

Fig. 2. SEM morphology of the (a) P1_MIP (b) P2_NIP
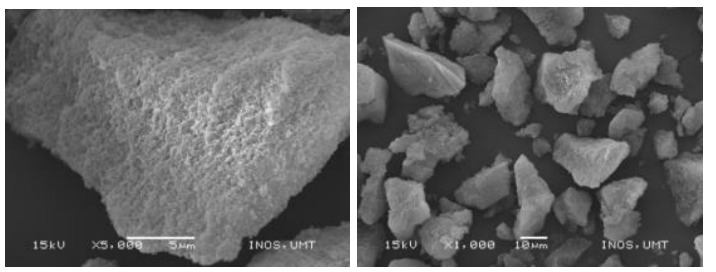

(a)
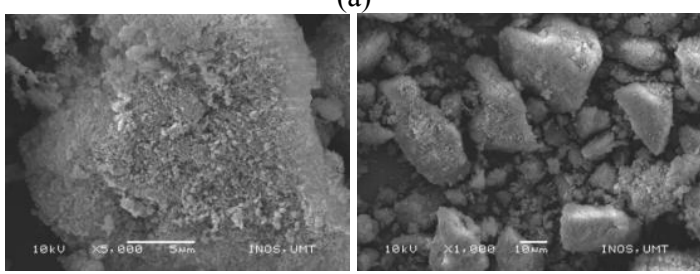

(b)

Fig. 3. SEM morphology of the (a) P3_MIP (b) P4_NIP

Porosimetry analysis has been done by BET technique based on the adsorption of gas onto the polymer surface. Table 2 is the summarized data from nitrogen sorption porosimetry experiments. The specific surface area for P3_MIP was slightly larger compared to P1_MIP. Moreover the average pore diameter in P3_MIP is also larger than P1_MIP, in which the polymer obtained was classified as mesoporous polymer (range between 2-50 nm) [12]. The larger the size of average pore diameter in P3_MIP might be due to the fact that less control during polymerization process. However, in the presence of RAFT agent, the morphology of the polymer could be controlled. This is the fact that the main equilibrium in RAFT polymerization process which leads to the pore volume smaller than in conventional polymerization. This also was in agreement with SEM results that showed rougher surface for P3_MIP with larger pore volume compared to P1_MIP.

Furthermore, the template molecule (thiamphenicol) is a small molecule $(<1 \mathrm{~nm})$ and aperture of pore is less than $4 \mathrm{~nm}$ in P1_MIP, it may be thought that the more effective adsorption capacity could be occured. On the other hand, the smaller size distribution could raise the selectivity and increase the imprinting factor value for the polymer [13].

Table 2. Nitrogen sorption porosimetry data for MIPs and NIPs prepared by RAFT polymerization and conventional FRP

\begin{tabular}{|c|c|c|c|}
\hline Samples & $\begin{array}{c}\text { Specific } \\
\text { surface area } \\
\left(\mathbf{m}^{2} \mathbf{g}^{-1}\right)\end{array}$ & $\begin{array}{c}\text { Total pore } \\
\text { volume } \\
\left(\mathbf{( c m}^{\mathbf{3}} \mathbf{g}^{-1}\right)\end{array}$ & $\begin{array}{c}\text { Average pore } \\
\text { diameter } \\
(\mathbf{n m})\end{array}$ \\
\hline P1_MIP & 335.94 & 0.3095 & 3.6849 \\
\hline P2_NIP & 326.05 & 0.5127 & 6.2900 \\
\hline P3_MIP & 340.33 & 0.9850 & 11.5770 \\
\hline P4_NIP & 273.58 & 0.9694 & 14.1740 \\
\hline
\end{tabular}

For adsorption/desorption isotherms studies, it was revealed more about the MIPs porosity characteristics. From the isotherm plots obtained, it was obviously showed that MIP/NIP synthesized using RAFT polymerization and conventional FRP having different isotherm types (Fig. 4 and Fig. 5). By comparing with isotherm types [14], the adsorption of thiamphenicol was significantly affected by the size of average pore diameter.

According to nitrogen adsorption/desorption isotherm and pore size distribution, P1_MIP and P2 NIP (Fig. 4) belong to Type IV isotherm. A Type IV isotherm is an indication of porous material containing micropores $(<2 \mathrm{~nm})$ and mesopores ( 2 to $50 \mathrm{~nm})$. It means that the polymer obtained having a formation of monolayer followed by multilayer. The concept of Type IV isotherm stated that at the low pressure end, monolayer adsorption and micropore filling occurs until the adsorption levels off as the micropores are filled. Then the mesopores continue filling by capillary condensation and once again adsorption levels off as the mesopores are filled. During desorption, as pressure is lowered, the mesopores are emptied by capillary evaporation, but when capillary condensation and capillary evaporation do not take place at the same pressure, a hysteresis loop is created [13, 15]. However, hysteresis loop obtained was much steeper for P2_NIP compared to P1_MIP. This might due to the template effect. 

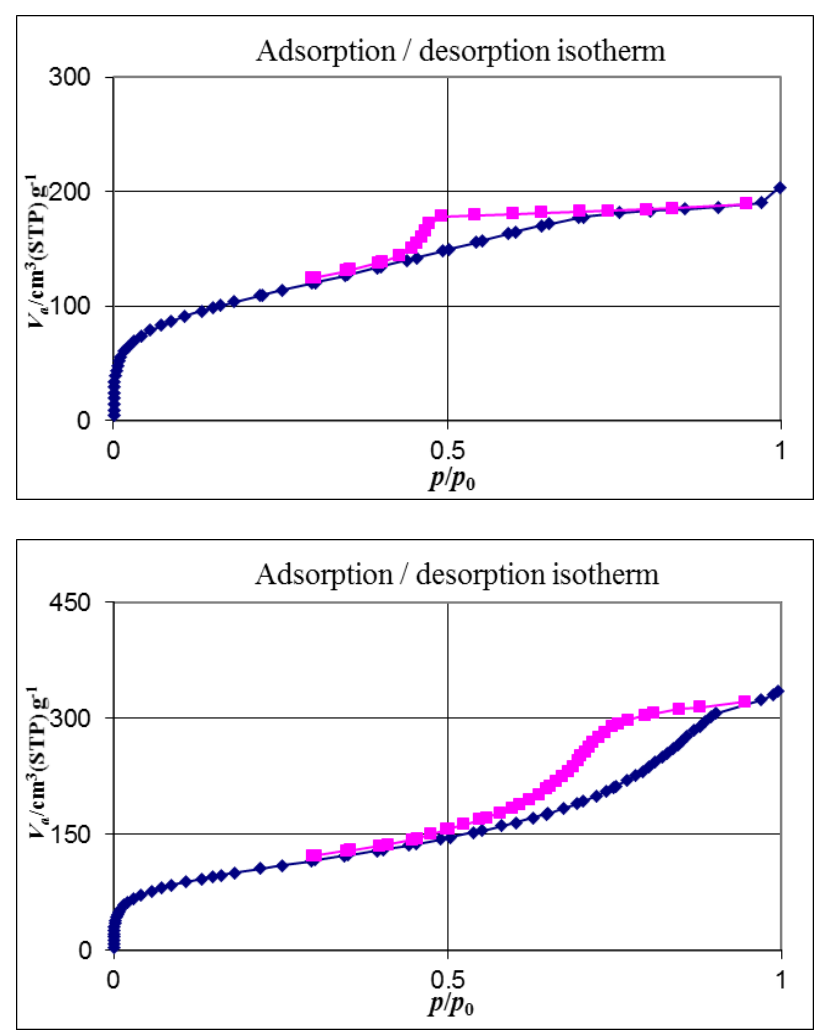

Fig. 4. Nitrogen adsorption/desorption isotherms obtained from measurement on P1_MIP (above) and P2_NIP (below) Blue : Adsorption

Pink : Desorption
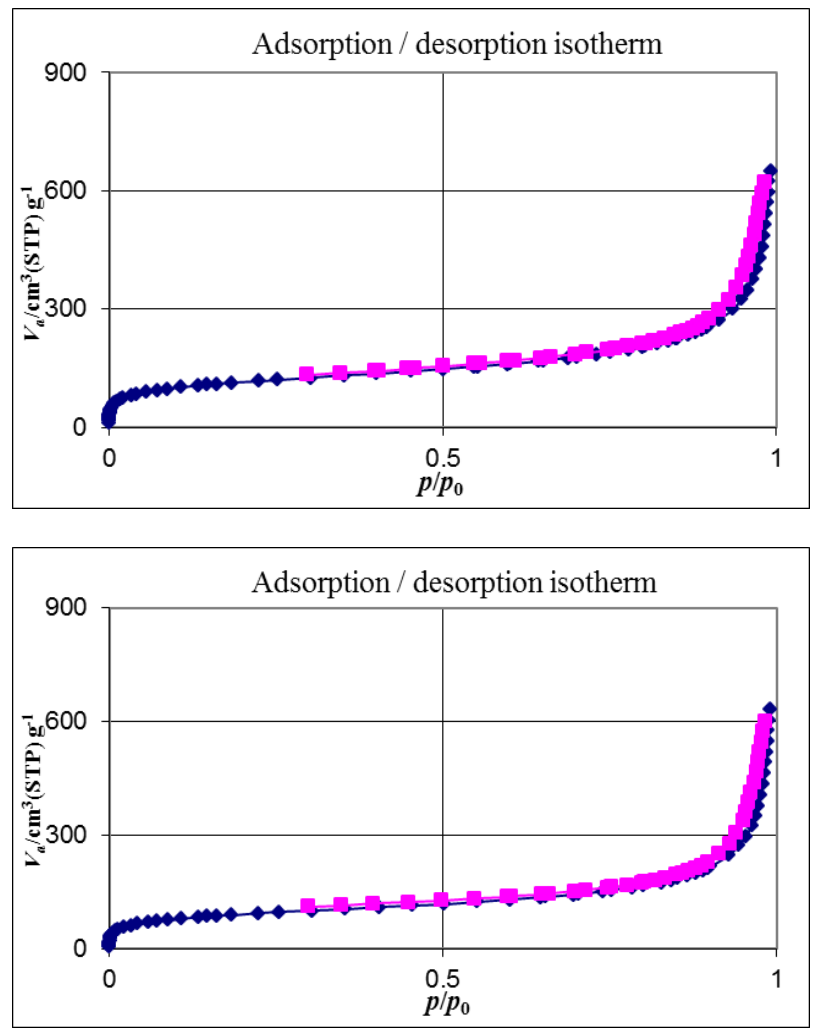

Fig. 5. Nitrogen adsorption/desorption isotherms obtained from measurement on P3_MIP (above) and P4_NIP (below) Blue : Adsorption Pink : Desorption
For polymers produced via conventional FRP (P3_MIP and P4_NIP), the isotherm type indicated belongs to Type III isotherm (Fig. 5). This isotherm also explains the formation of multilayer polymer and the adsorption/desorption of gas occurs in the same rate. This isotherm also explains adsorbed layer (surface of polymer) have a week interaction. From the plot, lack of knee could be represented extremely weak adsorbateadsorbent interaction. It was indicated that the polymers produced by conventional FRP having week interaction between them.

The monolithic polymers were characterized by using FTIR spectroscopy. The results showed that the P1_MIP/ P3 MIP and P2_NIP/P4_NIP have rather similar FTIR spectra (Fig. 6 and Fig. 7). As expected, it was because the same comonomers were used in the production of them. The presence of bands around $1738 \mathrm{~cm}^{-1}\left(\mathrm{C}=\mathrm{O}\right.$ ester stretch), $1228 \mathrm{~cm}^{-1}$ and $1217 \mathrm{~cm}^{-1}$ (C-O ester stretching) indicated the presence of EGDMA residues in the polymers produced. The signal at $1365 \mathrm{~cm}^{-1}$ showed to the $\mathrm{C}=\mathrm{C}$ stretch vibration peak from the pendant vinyl groups.

The imprinting process begins with complexation between MAA and thiamphenicol. The broadening at 3400$3500 \mathrm{~cm}^{-1}$ indicated that a hydrogen bonding interaction take place between hydroxyl group and amide group from thiamphenicol and carbonyl group from MAA residues. The FTIR spectra showed the absorption O-H and N-H stretch have been overlap each other. However, this signal more intense for both non-imprinted polymers, as this suggested that they have a lower cross-link density than MIPs. Unfortunately, the functional group $\mathrm{C}=\mathrm{S}$ and $\mathrm{C}-\mathrm{S}$ stretching derived from RAFT agent (CPDB) cannot be seen clearly in the FTIR spectra at the wavelength around $1050-1200 \mathrm{~cm}^{-1}$ might be due to only small amount of RAFT agent used. Moreover, since the particles obtained in irregular size and shape, some interaction sites were destroyed during grinding and thus give weak stretching absorption in FTIR spectra.

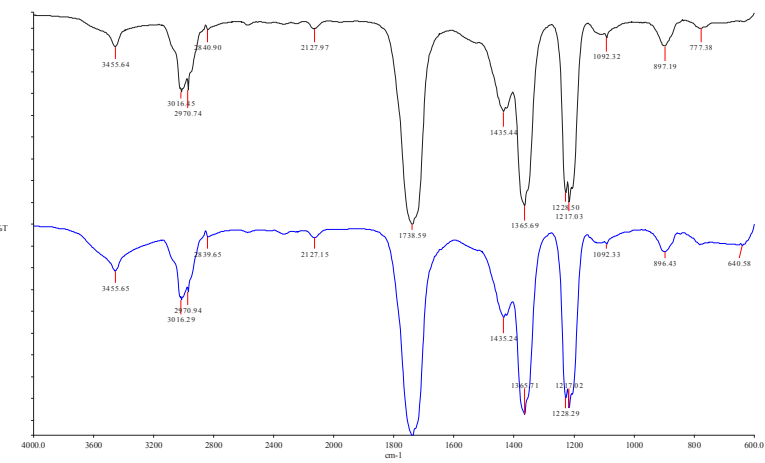

Fig. 6. FTIR spectra of P3_MIP (upper) and P1_MIP (below)

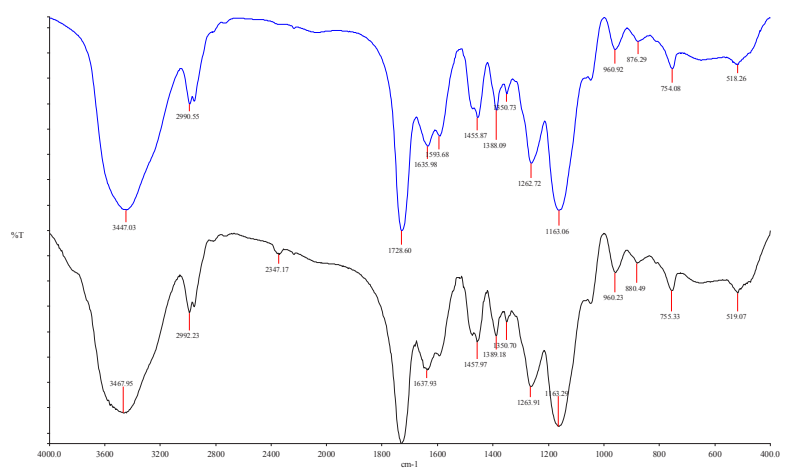

Fig. 7. FTIR spectra of P4_NIP (upper) and P2_NIP (below) 


\section{Conclusion}

The study was successfully demonstrated the production of thiamphenicol-imprinted polymers prepared by both RAFT and conventional free radical polymerization. This preliminary studies have suggested that additional benefits may arise from exploitation of the "living" character of controlled radical polymerization to produce MIPs. Further research will be focused on the adsorption studies of the polymers towards targeted molecule.

We would like to thank to Universiti Malaysia Terengganu for project funding.

\section{References}

1. B. Zu, Y. Zhang, X. Guo, H. Zhang, J. Polym. Sci., Part A: Polym. Chem., 48, 532-541 (2010)

2. C.J. Hawker, Acc. Chem. Res., 30, 373-382 (1997)

3. A. Goto, T. Fukuda, Prog. Polym. Sci., 29, 329-385 (2004)

4. J.-S. Wang, K. Matyjaszewski, J. Am. Chem. Soc., 117, 5614-5615 (1995)

5. J. Chiefari, Y. Chong, F. Ercole, J. Krstina, J. Jeffery, T.P. Le, R.T. Mayadunne, G.F. Meijs, C.L. Moad, G. Moad, Macromolecules, 31, 5559-5562 (1998)

6. D.G. Hawthorne, G. Moad, E. Rizzardo, S.H. Thang, Macromolecules, 32, 5457-5459 (1999)

7. S. Perrier, T.P. Davis, A.J. Carmichael, D.M. Haddleton, Eur. Polym. J., 39, 417-422 (2003)

8. G. Moad, Y. Chong, A. Postma, E. Rizzardo, S.H. Thang, Polymer, 46, 8458-8468 (2005)

9. P.A. Cormack, F.S. Mehamod, Sains Malays, 42, 529-535 (2013)

10. N.F. Yusof, F.S. Mehamod, N. Jusoh, K.A.M. Amin, F.B.M. Suah, AIP Conference Proceedings, 1885 (2017)

11. N.A. Othman, N.F. Yusof, R. Daik, F.S. Mehamod, International Journal of Technology, 8, 37-47 (2017)

12. F. Svec, T.B. Tennikova, Z. Deyl, Monolithic materials: preparation, properties and applications, (Elsevier, 2003)

13. S.-C. Lee, H. Chen, Polymer Journal, Vol. 41, No. 12, pp. 1092-1097, (2009)

14. J. Rouquerol, D. Avnir, C. Fairbridge, D. Everett, J. Haynes, N. Pernicone, J. Ramsay, K. Sing, K. Unger, Pure Appl. Chem., 66, 1739-1758 (1994)

15. J. Rouquerol, F. Rouquerol, P. Llewellyn, G. Maurin, K.S. Sing, Adsorption by powders and porous solids: principles, methodology and applications, (Academic press, 2013) 